\title{
Gibt es eine Lues nervosa? $\left.{ }^{1}\right)$
}

\author{
Von \\ Oskar Fischer.
}

(Aus der deutschen psychiatrischen Universitätsklinik in Prag.)

(Eingegangen am 4. März 1913.)

Der seit der kleinen und doch so bedeutungsvollen Publikation von Esmarch und Jesse ${ }^{2}$ ) aus dem Jahre 1857 entbrannte Kampf um die luetische Genese der progressiven Paralyse (später auch der Tabes dorsalis) dürfte wohl heute soviel wie entschieden sein, und zwar in dem Sinne, daß es ohne Lues keine Paralyse gibt. Schon dem Umstand, daß es auf einfachem anamnestischen Wege gelingt, bei etwa $70-90 \%$ der Paralytiker eine luetische Infektion als sicher oder sehr wahrscheinlich zu erweisen, muß eine ganz besondere Bedeutung beigelegt werden und der Einwand der Gegner der Luesätiologie der Paralyse, es gelänge doch nicht in allen Fällen, die luetische Infektion nachzuweisen, wird dadurch erledigt, daß, wie Hirschl zeigte, auch ein sehr großer Prozentsatz von tertiär Luetischen nichts von einer Primäraffektion weiß. Aber trotzdem wird die Ansicht vertreten, daß die Lues nicht die einzige Ursache der Paralyse sei, sondern daß ein, wenn auch vielleicht sehr geringer Teil der Paralysen durch andere Schädlichkeiten auch ohne Lues hervorgerufen werden könne. Die Erfahrungen mit der Wassermannschen Reaktion lassen aber eine derartige Auffassung nicht zu. Diese Reaktion ist bekanntlich (wenigstens für unsere Gegenden und gewisse seltene Ausnahmen nicht eingerechnet) nur bei der Lues und den metaluetischen Erkrankungen positiv; sie fällt aber nicht bei allen Luetikern positiv aus, sondern etwa bei $80 \%$. Wenn man sich auf den Standpunkt stellt, daß auch Nichtluetiker paralytisch werden können, so müßte man wohl erwarten, daß ein gewisser Teil der Paralytiker serologisch ebenfalls negativ reagieren müßte, und zwar die $10 \%$, bei denen auch die genaueste Anamnese keine Infektion ergeben kann. Da aber bei manifester Lues nur etwa $80 \%$ positiv reagieren, könnten nur $80 \%$ von $90 \%$, das sind $74 \%$

1) Vortrag, gehalten in der ,Wissenschaftlichen Gesellschaft deutscher Ärzte in Böhmen" in Prag am 28. II. 1913.

2) Allgem. Zeitschr. f. Psych. 14. 
positiv sein. Demgegenüber haben aber die bereits sehr zahlreichen Erfahrungen einer großen Anzahl von Autoren (Pla ut u. a.) ergeben, daß sich bei der Paralyse beinahe ausnahmslos, also etwa in $100 \%$. eine positive Serumreaktion ergibt.

Wenn wir auch heute die biologischen Grundlagen der Wassermannschen Reaktion noch nicht kennen, so kann man dennoch so viel bereits als feststehend hinnehmen, daß dieselbe, kaum in Betracht kommende Ausnahmen nicht eingerechnet, nur dann auftritt, wenn eine syphilitische Infektion vorausgegangen ist. Demnach müssen wir zum Schlusse gelangen, daß alle Paralytiker eine luetische Infektion überstanden haben. Hiermit ist jedenfalls auch gesagt, daß die Lues der Hauptfaktor ist, die Frage jedoch, ob auch der einzige Faktor, ist damit nicht erledigt. Denn die Umkehrung der Luesparalyse (Tabes)Statistik im Sinne der Frage: Wieviel Luetiker erkranken an der Metalues? ergibt, daß nur ein ganz verschwindender Bruchteil der Luetiker metaluetisch wird. Aus selbstverständlichen Gründen ist es sehr schwer, ein entsprechend großes Material (und ein sehr großes Material ist dazu wegen der sonst enormen Zufallsfehlerquellen erforderlich) in jeder Richtung einwandsfrei zusammenzustellen, weswegen auch bisher nur wenig derartige zahlenmäßige Feststellungen existieren.

Matthes ${ }^{1}$ ) berechnete bei 698 mit Lues infizierten Personen eine Morbiditätsziffer für Paralyse von $1-2 \%$.

Ph. J. Pick und Bandler ${ }^{2}$ ) bearbeiteten katamnestisch die in der Prager dermatologischen Klinik in den Jahren 1879-1899 behandelten Luetiker (Abschluß der Arbeit 1904). Es ließ sich das Schicksal von 2066 Fällen eruieren, von denen 28 an Paralyse erkrankt sind, was einem Prozentsatz von 1,3 entspricht. Die Statistik von Pick und Bandler hat den Nachteil, daß Männer und Frauen summarisch und nicht gesondert behandelt werden, was deshalb unzweckmäßig ist, weil schon nach der rein klinischen Erfahrung Frauen unverhältnismäßig seltener an Paralyse erkranken als Männer; man möchte also schon deshalb andere Prozentverhältnisse für die beiden Geschlechter erwarten.

Ich habe deshalb in dem Material von Pick und Bandler die Männer und die Frauen gesondert, wobei sich ganz gewaltige Differenzen ergaben: auf 1178 Männer kommen hier 25 Paralysen, d. i. 2,1\% und 12 Tabesfälle, das entspricht $1,6 \%$, in Summa an Metalues 3,7\%; von 888 Frauen erkrankten dagegen nur 3 an Paralyse, was $0,33 \%$ entspricht und 2 an Tabes, d. i. 0,22\%, in Summa an Metalues nur 0,55\%. Wir haben also hier den statistisch zahlenmäßigen Beweis, daß die

i) Statistische Untersuchungen über die Folgen der Lues. Münch. med. Wochenschr. 1902.

2) Archiv f. Dermatol. u. Syphilis. 101. 1910. 
Metalues bei luetischen Frauen etwa 7 mal seltener auftritt als bei luetischen Männern.

Zu wesentlich höheren Ziffern gelangten Matta uschek und Pilcz ${ }^{1}$ ), welche das Schicksal von 4134 luetisch erkrankten Offizieren nach einer entsprechenden Reihe von Jahren verfolgen konnten, von Offizieren, deren Lues militärärztlich behandelt worden war und deren Krankengeschichten vorliegen. Dieses Material ist deswegen besonders wichtig und kostbar, weil man dabei mit viel größerer Sicherheit und viel weniger Verlustfehlerquellen das Schicksal der Erkrankten verfolgen konnte; als Prozentsatz fand sich hier für die Paralyse 4,67 und für die Tabes 1,6, also in Summa für die Metalues 6,27\%. Die so gefundene hohe Ziffer differiert recht beträchtlich von denen der anderen Autoren, von $1-2 \%$ von Matthes und $1,3 \%$ von Pick und Bandler; sie differiert aber viel weniger von der Ziffer der aus der Pick - Bandlerschen Statistik berechneten männlichen Metaluesmorbidität von 3,6\% Die immer noch bestehende Differenz könnte einerseits in den geringeren Fehlerquellen bei Mattauschek und Pilcz ihren Grund haben, andererseits könnte sie auch darauf zurückgeführt werden, daß es sich hierbei um Offiziere handelt, deren Lebensführung auch sonst eine schwere Schädigung des Nervensystems mit sich bringt. Doch möchte ich hervorheben, daß die Tabesmorbidität bei Pick und Bandler, ebenso wie bei Mattauschek und Pilcz 1,6\% beträgt, welchem Umstande, vorausgesetzt daß nicht ein Zufall mitspielt, auch eine entsprechende Bedeutung zukommt.

Die Gründe, warum der eine Luetiker metaluetisch wird, der andere nicht, sind uns noch nicht vollkommen bekannt und klar. Man hat in erster Linie noch andere Ursachen (d. i. Schädigungen) angenommen, welche mit der Lues (ev. nicht gleichzeitig) das Individuum treffen, und welche bei entsprechender Konstellation erst die Form der Erkrankung bestimmen. Dafür könnten gerade die hier angeführten Prozentverhältnisse einen Beweis liefern; wenn man bei gleichbleibendem Prozentsatz der Tabesmorbidität die Paralysemorbidität der Offiziere um mehr als das Doppelte größer findet als die der Zivilisten, so kann man dies doch in gewisser Hinsicht als einen zahlenmäßigen Beweis für diese Ansicht hinstellen, zumal man bisher immer nur von allgemeinen Eindrücken sprechen konnte, von denen erfahrene Psychiater berichtet haben.

Außerdem ergeben sich noch Gründe für die Auffassung, daß es eine besondere Lues nervosa, resp. wie die Franzosen sich ausdrücken „Syphilis à virus nerveux" gibt, d.h. um mit den Worten Erbs") zu sprechen ,Formen der Syphilis, welche mit ihrer Schädigung mit

1) Zeitschr. f. d. ges. Neur. u. Psych. 8.

2) Deutsche Zeitschr. f. Nervenheilk. 1905. 
Vorliebe das Nervensystem heimsuchen, deren Krankheitserreger selbst oder vermittels der von ihnen erzeugten Blutmischung (Toxine, Antitoxine usw.) gerade auf die nervösen Elemente eine besonders schädliche Wirkung ausüben".

$\mathrm{Zu}$ dieser Ansicht führten in erster Linie die immer mehr gehäuften Beobachtungen von Metalues bei noch nicht geschlechtsreifen Kindern luetischer Eltern, die aber selbst nie Symptome von Metalues aufzuweisen hatten.

Bei diesen jugendlichen Individuen konnte man die andern als ätiologischen Momente angesehenen Schädlichkeiten: wie Alkoholismus, Uberanstrengung, psychische und physische Traumen, geschlechtliche Ausschweifungen ausschließen; es blieb also nur die Lues übrig. Man mußte deshalb in diesen Fällen die Lues allein, ohne die andern Momente, als Ursache der Paralyse oder Tabes auffassen, und diese Form der Lues, welche allein und als solche zur Metalues führt, wurde zur Lues nervosa.

Allmählich wurde man auch auf die sich mehrenden Fälle von konjugaler Paralyse resp. Tabes aufmerksam. Wenn zwei nicht blutsverwandte Ehegatten, bei denen die Heredität demnach kaum eine Rolle spielen kann, die aber beide (und zwar einer vom anderen) syphilitisch wurden, in gleicher Art metaluetisch werden, dann muß bereits die Lues als solche die Ursache der Metalues darstellen. Die Übertragung der gleichen ,Luesart" mit der gleichen Affinität zum Nervensystem mußte denn auch zur Ansicht führen, daß die Lues nervosa als solche überimpfbar und übertragbar ist. Für die letztere Auffassung scheinen aber besonders die wenn auch seltenen, aber um so merkwürdigeren Beobachtungen zu sprechen, die sich auf mehrere an gleicher Quelle infizierte Luetiker beziehen, die insgesamt metaluetisch wurden. Die wichtigsten dieser Beobachtungen sind folgende:

Mendel: Ein luetischer Mann infizierte seine Frau; er selbst starb an Paralyse, die Frau heiratete wieder, infizierte ihren zweiten Mann, und sie und der zweite Mann wurden tabisch.

R égis: Ein Mann infizierte seine Frau und Schwägerin mit Syphilis; alle 3 wurden paralytisch.

Nonnes Patient bekommt 6 Jahre nach der Infektion eine Tabes und die Person, von der er sich angesteckt und mit der er nicht mehr zusammenlebte, wird nach 8 Jahren tabisch.

Nonne: Ein von einem Fremden infiziertes Kind wird tabisch; das Kind hat seine Mutter und diese wieder ihren Mann, den Vater des Kindes infiziert und beide Eltern wurden tabisch.

Nonne: 3 untereinander befreundete Männer haben sich in einer Nacht von einer syphilitischen Puella angesteckt; einer derselben wurde tabisch, die beiden anderen paralytisch. 
Erb: 5 untereinander nicht verwandte Männer infizieren sich von derselben Person und alle werden später tabisch resp. paralytisch.

Brosius: 7 Glasbläser wurden von einem Kameraden vermittels der Glaspfeife im Munde syphilitisch infiziert; alle bekommen einen typischen Lippenschanker; von diesen 7 konnten später 5 untersucht werden; 2 davon hatten Tabes und 2 Paralyse.

Junius und Arndt: Ein Mann, der 2 mal verheiratet war, wurde paralytisch; seine 24 jährige Tochter starb an Paralyse und seine beiden Frauen bekamen Tabes.

In ähnlicher Weise versuchte man später auch die immer mehr sich häufenden Beobachtungen von Paralyse bei Kindern, deren Vater oder Mutter oder beide an Metalues erkrankt waren, zu erklären.

Alle diese Beobachtungen, so bemerkenswert sie auch sind, können aber nicht als zwingender Beweis für die Existenz einer Lues nervosa gelten, was im allgemeinen auch von den Anhängern der Lues nervosa zugegeben wird. So konnte man mit Recht darauf hinweisen, daß gerade die Fälle von hereditärer Paralyse in keiner Weise als Argumente für die Lues nervosa gelten können, weil es recht wahrscheinlich erscheint, daß das Kind eines Paralytikers neben einer Lues auch noch eine hereditäre degenerative Anlage seines Nervensystems mit auf die Welt bringt, und daß eben diese zwei Momente viel eher und leichter eine Paralyse hervorrufen können, als die Lues allein. Viel schwerer fallen ins Gewicht die Beobachtungen von mehreren Metaluesfällen, welche demselben Infektionsherd ihre Lues verdanken, doch auch hier ist der Einwand nicht von der Hand zu weisen, daß solche doch immerhin vereinzelt dastehende Beobachtungen bei der kolossalen Zahl von verschieden durcheinander gewürfelten Luesfällen auch als reine $\mathrm{Zu}$ fälle sich ergeben können, genau so wie unter Tausenden von Möglichkeiten der reine Zufall die einzelnen Nummern einer Lotterie zu einem voraus gewählten Terno herauskommen läßt. Derselbe Einwand gilt auch für die konjugale Paralyse; auch diese könnte auf rein zufälliger Zusammenwürfelung basieren. Ein Beweis für das Vorhandensein oder Nichtvorhandensein einer Lues nervosa wird eben erst dann geliefert werden können, wenn es gelingen würde, rein statistisch, - durch genauen und einwandfreien Vergleich, - nachzuweisen, daß unter einer großen Zahl von an einem bestimmten Herd infizierten Luetikern prozentuell viel mehr Metaluesfälle sich entwickeln als bei anderen. Es ist von vornherein recht unwahrscheinlich, daß ein derartiger Beweis gelingen könnte.

Ich habe versucht, für diesen Beweis auf einem andern Wege eine Basis zu finden.

Man weiß nämlich, wie eingangs besprochen wurde, mit welcher Wahrscheinlichkeit ein Luetiker Metalues bekommt, d. h. wieviel Pro- 
zent der Luetiker metaluetisch werden. Wenn es nun richtig wäre, daß die Hauptursache der Paralyse eine besonders geartete luetische Infektion ist, dann müßte sich folgende Möglichkeit ergeben: Gesetzt den Fall, man hätte 100 verheiratete Paralysen und hätte Grund anzunehmen, daß die Ehehälften derselben ebenfalls luetisch erkrankt waren, dann muß unter diesen auch ein bestimmter Prozentsatz paralytisch werden. Wenn dieser Prozentsatz dem sonst bekannten Prozentsatz entspricht, dann ist die Lues nervosa ganz unwahrscheinlich; wenn sich aber bei genügend großem Material ein wesentlich höherer Prozentsatz ergeben würde, dann wäre dies ein recht wichtiger Beweis für deren Existenz.

Bei einem zu andern Zwecken statistisch verarbeiteten Material von klinisch beobachteten 500 Paralysen habe ich die Fälle auch von diesem Gesichtspunkte aus zusammengestellt.

Mein Material bestand aus 407 Männern und 93 Frauen. Von diesen waren 319 Männer und 76 Frauen verheiratet. Auf diese 395 Fälle verheirateter Paralysen kommen nur 10 Fälle von konjugaler Metalues, was dem Prozentsatz von $2,53 \%$ entspricht. Dies ist ein so niedriger Prozentsatz, daß man sich auf den ersten Anblick berechtigt fühlen könnte, die ganze Frage als im negativen Sinne erledigt anzusehen. Die Sache verhält sich aber ganz anders, wenn man die Frauen und Männer gesondert betrachtet. Dann ergibt sich folgendes:

Bei den 319 Männern ließ sich eruieren, daß nur 3 mal die Frau eine Metalues hatte, und zwar 1 mal Paralyse und 2 mal Tabes. Unter den 76 Frauen gab es aber 7, deren Männer eine Metalues hatten, und zwar 5 Paralyse und 2 Tabes. Prozentual berechnet würde die konjugale Metalues (Paralyse und Tabes) bei den Frauen paralytischer Männer nur 0,94\% entsprechen, und bei den Männern paralytischer Frauen den hohen Prozentsatz von 9,2\% darstellen.

Es ist wohl klar, daß der so gefundene Prozentsatz von $0,94 \%$ konjugal-metaluetischer Frauen von paralytischen Männern nicht berücksichtigt werden kann, und zwar aus folgenden Gründen:

1. Man weiß nicht, wie viele von den Frauen der paralytischen Männer auch infiziert waren; denn die Mehrzahl der Männer holt sich ihre Lues lange vor ihrer Ehe und bei einem wohl nicht unbeträchtlichen Teil verliert die Lues ihre Infektiosität.

2. Da die Mehrzahl der Frauen von Paralytikern von ihren Männern in der Ehe infiziert werden, ist die Lues bei den Frauen der Paralytiker wesentlich jüngeren Datums als die der Männer. Man muß also schon aus diesem Grunde als recht wahrscheinlich annehmen, daß die Frauen von Paralytikern eigentlich später ihre Metalues bekommen wie die Männer, was ja schließlich, zu einem gewissen Teile wenigstens, auch die Statistik der konjugalen Paralyse ergibt. Man könnte ein der- 
artiges Material also erst dann verwenden, wenn man in der Lage wäre, etwa 10 Jahre später das Schicksal der Frauen der männlichen Paralysen zu verfolgen.

3. Die weibliche Paralyse ist überall wesentlich seltener als die der Männer, und zwar viel seltener, als es der geringer verbreiteten Lues bei den Frauen entspricht; deswegen darf als Vergleichszahl nicht die allgemein gefundene Morbiditätsziffer der Metaluetiker gelten, sondern die Prozentzahl, welche sich bei Berechnung weiblicher Luetiker allein ergibt, d. i. nach Umrechnung des Materials von Pick und Bandler $0,55 \%$.

Trotz dieser vielen Fehlerquellen ergibt sich bei unserem konjugalen Materiale der doch immerhin höhere Prozentsatz von 0,94.

Ganz andere Verhältnisse ergeben sich aber bei der Betrachtung der zweiten Gruppe, wobei prozentual festgestellt wurde, wie viele von den Ehemännern paralytischer Frauen metaluetisch werden. Dabei können wir bereits annehmen, daß der größte Teil der an Metalues erkrankten Frauen ihre Lues von den Männern in der Ehe akquirierte; zwar ist es nicht ganz wahrscheinlich, daß dies für alle zutrifft, immerhin aber wohl für einen sehr hohen Prozentsatz. Die Männer dieser Frauen, welche meist viel früher ihre Lues akquiriert haben, werden demnach im allgemeinen auch früher als ihre Frauen der Metalues verfallen, was mit Leichtigkeit auch bei gerade nicht sehr genauer Anamnesenführung eruiert werden kann.

Schließlich lassen sich diese Prozentverhältnisse mit den bei ,gewöhnlichen" Luetikern gefundenen besser vergleichen, weil man dabei meist (Pilcz und Mattauschek nur) Männer berücksichtigt hatte.

Da aber bekanntlich die Paralysemorbidität in verschiedenen Gegenden und zu verschiedenen Zeiten auch verschieden ist, so ist es klar, daß man bestrebt sein muß, ein gleichartiges Material, sowohl was Ort der Beobachtung als auch Zeit anbelangt, nebeneinanderzustellen.

Mein Beobachtungsmaterial besteht aus Fällen der Prager psychiatrischen Klinik aus den Jahren 1902-1910. Da im Durchschnitt diese Fälle ihre Lues vor 15 Jahren akquiriert haben, ist die Mehrzahl dieser Fälle in den Jahren 1887-1895 infiziert gewesen. Einem glücklichen Zufalle habe ich es nun zu verdanken, daß Pick und Bandler dem Schicksal eines sehr großen Materials vop Luetikern katamnestisch nachgeforscht haben, die derselben Bevölkerungsklasse, demselben Teile des Landes entstammen und etwa auch zu derselben Zeit infiziert gewesen waren. Pick und Bandler fanden, wie schon erwähnt wurde, bei Männern 3,7\% von Metalues, wogegen sich bei unserem Material die hohe Zahl von $9,2 \%$ ergibt; dabei muß diese Zahl wohl mehr wie alle anderen eine Minimalzahl sein. Denn vorerst muß betont werden, daß nicht angenommen werden kann, daß alle Männer unserer Frauen- 
paralysen auch luetisch waren, weiter wissen wir, daß zwar im allgemeinen die Männer bei den konjugalen Paralysen früher erkranken als die Frauen, aber nicht durchwegs; denn nach dem Material von Junius und Arndt und dem sonst in der Literatur erwähnten, erkrankt die Frau nur in etwa $1 / 4$ der Fälle früher, in $3 / 4$ der Mann früher oder beide zugleich. Wir könnten also deshalb schließen, daß unser berechneter Prozentsatz um $1 / 3$, das ist also auf etwa $12 \%$ sich erhöhen müßte. Ich bin deshalb dem Schicksal der Männer der paralytischen Frauen einstweilen insoweit nachgegangen, als ich nachsah, ob nicht irgendeiner derselben nachträglich als Paralytiker in die Irrenanstalt aufgenommen wurde; bei diesen Nachforschungen fand sich noch ein Fall vor, so daß trotz der immer noch nicht ganz vollständigen ${ }^{1}$ ) Erhebungen auf 76 verheiratete weibliche Paralysen 8 metaluetische Ehemänner entfallen, was einem Prozentsatz von $10,5 \%$ entspricht.

Diese besonders große Differenz - 3,7\%: 10,5\% - spricht eben dafür, daß diejenigen Personen, welche von später an Paralyse erkrankenden Luetikern angesteckt werden, viel mehr gefährdet sind, eine Metalues zu bekommen als andere Luetiker, was einem zahlenmäßigen Beweis für die Lues nervosa" gleichkommt.

Doch bei Statistiken muß man bekanntlich mit den Schlußfolgerungen immer sehr vorsichtig sein, vielleicht viel vorsichtiger als sonst, da der Zufall sich nicht so ohne weiteres ausschließen läßt. Deshalb habe ich auch nach einem Material anderer Autoren gesucht, das so zusammengetragen wäre, daß man es auch für unsere Frage verwenden könnte. In einwandsfreier Weise ließ sich dazu die Arbeit von $\mathrm{Kron}^{2}$ ): „Tabes dorsalis beim weiblichen Geschlecht" verwenden.

Kron hat 160 tabische Frauen untersucht, von denen 117 verheiratet waren. 12 Frauen hatten metaluetische Männer, und zwar waren die Erkrankungen so verteilt, daß bei 6 Ehepaaren beide Ehegatten Tabes liatten und bei 6 Ehepaaren die Frau Tabes und der Mann Paralyse. Angenommen, daß alle Männer dieser tabischen Frauen luetisch waren, so entfällt auf diese Männer der Metalues-Prozentsatz (117:12) von $10,2 \%$.

Daß man bei der Tabes und einem Material aus anderen Verhältnissen ganz ähnliche Prozentverhältnisse erhält, ist wohl mehr als Zufall.

Ich habe überdies noch aus der Arbeit von Mönkemöller ${ }^{3}$ ) auf

1) Man hätte dem Schicksal der Ehemänner noch etwas genauer nachgehen müssen, dann hätte sich die Prozentzahl vielleicht um 1-2\% erhöht; die auf dem hier eingeschlagenen Wege erhobene Prozentdifferenz ist aber so groß, daß sie zur Beweisführung vollkommen genügt.

2) Deutsche Zeitschr. f. Nervenheilk. 1898.

3) Monatsschr. f. Psych. u. Neurol. y. 
ähnlicher Basis berechnete Prozentverhältnisse zu erzielen versucht. Mönkemöller berichtet nämlich, daß er unter 741 Paralysen aus dem Materiale von Herzberge $18 \mathrm{mal}$ konjugale Metalues (d. h. 18 Paare) beobachtet hatte:

14 mal waren beide Ehegatten paralytisch,

2 mal der Mann tabisch, die Frau paralytisch,

1 mal der Mann paralytisch, die Frau tabisch,

1 mal der Mann tabisch, die Frau tabisch.

18 Fälle von 741 entsprechen einem Prozentsatz von $2,42 \%$, was wieder recht gut mit meinem summarischen Prozentsatz von 2,35 überein. stimmt.

Natürlich kann man daraus noch nichts entnehmen, da Männer und Frauen, und zwar die verheirateten gesondert berechnet werden müssen. Nun macht aber Mönkemöller keine Angaben darüber. In der Arbeit von Junius und Arndt'), die das Material von Dalldorf (also ebenfalls Berliner Bevölkerung) bearbeitet haben, sind die prozentuellen Verhältnisse der paralytischen Männer und Frauen berechnet, sowie auch das Verhältnis der Ledigen zu den Verheirateten.

Wenn man nach diesem Verhältnis die 741 Paralysen Mönkemöllers zergliedert, so kommt man auf etwa 172 verheiratete Frauen. Unter den 18 Metalues-Ehepaaren waren 16 Frauen paralytisch, es hatten also von 172 (von mir berechnete Wahrscheinlichkeitsziffer) verheirateten paralytischen Frauen 16 metaluetische Männer, das entspricht $9,3 \%$.

Es ist ja zuzugeben, daß diese Schlüsse aus dem Materiale Mönkemöllers nicht bindend sein können, sie gewinnen aber bei der übereinstimmenden Prozentzahl eine gewisse Bedeutung.

Gegen alle diese Berechnungen kann aber ein sehr gewichtiger Einwand gemacht werden.

Es ist klar, daß das Material einer dermatologischen Klinik nicht ohne weiteres den allgemeinen Zahlenverhältnissen der luetisch infizierten Bevölkerung entspricht. Wohl die allermeisten Luetiker, welche auf einer dermatologischen Klinik sich vorstellen, werden antiluetisch behandelt, obzwar nicht immer eine gründliche Quecksilberkur durchgeführt werden kann. Dagegen kann man aber mit Recht annehmen, daß die Männer unserer paralytischen Frauen jedenfalls in viel höherem Maße ohne Behandlung gewesen sind als unser Vergleichsmaterial, die Fälle der dermatologischen Klinik, welche Pick und Bandler verarbeitet haben. Man könnte also demnach einwenden, daß die große Differenz sich nur deshalb ergibt, weil die Männer der paralytischen Frauen verhältnismäßig weniger oder schlechter antiluetisch behandelt wurden als das Vergleichsmaterial.

1) Archiv f. Psych. 44. 
Wir müssen uns demnach, um unsere Frage sicher entscheiden zu können, auch mit der Frage befassen, ob wir denn aus anderweitigen Beobachtungen tatsächlich genügende Anhaltspunkte für die Ansicht besitzen, daß die antiluetische Behandlung die Entwicklung einer Metalues verhüten kann.

Die Literatur zeigt uns, daß in diesem Punkt noch Unklarheit und Widerstreit der Meinungen herrscht.

Nach Fournier, der bei seinem Material (82 Paralysen) gefunden hat, daß nur 5\% der Kranken gut antiluetisch behandelt wurden, wogegen bei $15 \%$ nur eine mittelmäßige und $80 \%$ eine ganz ungenügende Therapie durchgeführt worden war, ist das wichtigste Prophylaktikum der Metalues eine gründliche Behandlung der Lues; ähnlich äußert sich Neisser, in dessen Material von Tabesfällen ein besonders großer Prozentsatz von nur ungenügend oder gar nicht antiluetisch Behandelten sich befand.

Zu ganz anderen Resultaten kommt aber Collins, Schuster ${ }^{\mathbf{1}}$ ) und $\mathrm{Kron}^{2}$ ), nach denen die antiluetische Therapie (gemeint ist nur die Quecksilberbehandlung) keinen günstigen Einfluß hinsichtlich Verhütung der Metalues auszuüben imstande ist, ja es zeigte sich sogar nach den Berechnungen von Schuster und Kron, daß bei behandelten Luetikern die Metalues um eine Spur früher auftritt als bei Nichtbehandelten.

Auch für diese noch strittige Frage bringt uns die bereits erwähnte Arbeit von Mattauschek und Pilcz ein ganz besonders wertvolles Material. Die Autoren haben ihre Fälle auch vom Standpunkte dieser Frage gesichtet und gelangen zu dem Schlusse, daß, ,wenngleich auch eine sehr gründliche Behandlung der Syphilis vor späterer Paralyse nicht schützt, der Vergleich einer Serie von Syphilitikern mit mangelhafter Therapie und einer Serie von chronisch-intermittierend Behandelten zu ergeben scheint, daß der Prozentsatz der davon später paralytisch Gewordenen ein wesentlich geringerer bei den letzteren ist."

Aus der Tabelle IV von Mattauschek und Pilez ist ersichtlich, daß die Paralyse sich nach ihrem Material entwickelt:

bei Luetikern ohne spezifische Behandlung in $23,23 \%$,

bei Luetikern mit einmaliger $\mathrm{Hg}$-Kur in $30,61 \%$,

bei Luetikern mit wiederholter energischer Behandlung in 3,47\%. Diese Tabelle ergibt also ganz ähnliche Prozentverhältnisse wie das Material von Fournier; Mattauschek und Pilcz bemerken aber dabei mit Recht, daß man immerhin unterscheiden müsse zwischen rezidivfreien und rezidivierenden Luesfällen, wobei gewiß aus natürlichen Gründen die rezidivfreien eine weniger gründliche Kur durch-

1) Vers. des Vereines deutscher Nervenärzte; Dresden 1907.

2) l. c.

Z. f. d. g. Neur. u. Psych. O. XVI. 
gemacht haben werden als die andern. Um dieses gegenseitige Verhältnis klarzulegen, haben Mattauschek und Pilcz die hier beigefügte Tabelle XI zusammengestellt und schließen daraus, daß man deshalb nicht berechtigt sei zum Schluß, ,daß ein durch energische Therapie bedingtes Zurückdrängen der äußerlichen Syphilisrezidive mit Rücksicht auf spätere Nervenerkrankungen ungünstig sein könnte." Vielmehr ergebe sich daraus, daß die rezidivfreien Luetiker auch trotz mangelhafter Therapie von ihrer Lues dauernd geheilt bleiben.

Tabelle XI von Mattauschek und Pilcz. Verhältnis der Rezidive und Behandlung bei Paralysen.

\begin{tabular}{c|c|c|c|c}
\hline \hline Rezidive & $\begin{array}{c}\text { Keine oder nur } \\
\text { Lokalbehandlung }\end{array}$ & $\begin{array}{c}\text { Eine einzige } \\
\text { Hg-Kur }\end{array}$ & $\begin{array}{c}\text { Mehrfache } \\
\text { Hg-Kuren }\end{array}$ & Summe \\
\hline$\theta$ & 33 & 44 & 6 & 83 \\
1 & 2 & 8 & 19 & 29 \\
mehrere & 3 & 4 & 27 & 34 \\
\hline Summe & 38 & 56 & 52 & 146
\end{tabular}

Mit diesem Schlusse möchte ich mich aber nicht ganz begnügen. Wir müssen bei dieser Frage in erster Linie die bereits zur Regel gewordene Erfahrung berücksichtigen, daß die meisten Paralytiker nur eine leichte Lues gehabt haben, $d . h$. eine Lues, die nur als ein kleiner Primäraffekt zutage tritt, im besten Falle noch ein ganz vorübergehendes Exanthem entwickelt, später aber keine manifesten Symptome mehr zeigt. Es ist klar, daß derartige Kranke viel seltener antiluetisch behandelt werden als solche, die unter mehrfachen oder gar schweren Rezidiven zu leiden haben, wobei wieder zu bemerken ist, daß es zu den größten Seltenheiten gehört, wenn eine schwere Lues in eine Paralyse ausgeht. So bemerkt Fournier, daß sich unter 245 Fällen von schwerer Lues keine einzige Paralyse entwickelt hatte, wohl aber viele davon an Lues cerebri erkrankt sind. Deshalb ist ja auch wiederholt die Meinung ausgesprochen worden, daß gerade die leichte Lues zu Paralyse führt. Besonders lehrreiche Zahlen ergeben sich gerade in der Arbeit von Matta uschek und Pilcz; von 87 Luetikern mit leichter Lues ohne Rezidive sind 37, d. i. $42,53 \%$ an Paralyse erkrankt, wogegen von 1778 Luetikern mit Rezidiven nur 49 paralytisch wurden, was dem Prozentsatz von nur 2,53 entspricht. Man hat diesen merkwürdigen Umstand auch damit zu erklären versucht, daß die leichten Luesfälle nur deshalb metaluetisch werden, weil sie in ungenügender Weise antiluetisch behandelt worden sind. Ob diese Ansicht berechtigt ist, soll folgende Utberlegung zeigen.

Zwei gewichtige und grundverschiedene Momente sind es, die sich hier gegenüberstehen; auf einer Seite steht die ,leichte Lues" mit der fraglichen Eigenschaft, Metalues zu erzeugen, auf der andern Seite die. 
mangelhafte Therapie. Die Entscheidung der Frage, welchem dieser zwei Momente eine größere Bedeutung für die Paralyse zufällt, läßt sich nur auf statistischem Wege erweisen, und zwar bei Berücksichtigung des gegenseitigen Verhältnisses zwischen gründlicher und schlechter Behandlung einerseits und leichten und schweren Luesfällen andererseits. Ein derartiges Material ergibt die Tabelle XI von Matta uschek und Pilcz. Ich würde aber aus dieser Tabelle eine andere Antwort herauslesen als die genannten Autoren. Wenn wir nämlich die Tabelle, die sich auf 146 Paralysen bezieht, aus Gründen besserer Ubbersichtlichkeit zusammenziehen, so ergeben sich folgende zwei Vereinfachungen:

Vereinfachung A der Tabelle XI von Mattauschek und Pilcz.

\begin{tabular}{|c|c|c|c|c|c|}
\hline Rezidive & $\begin{array}{l}\text { Unge } \\
\text { oder } \\
\text { Beh }\end{array}$ & $\begin{array}{l}\text { ügende } \\
\text { ine Hg- } \\
\text { udlung } \\
\text { in Pro- } \\
\text { zenten }\end{array}$ & & $\begin{array}{l}\text { fache } \\
\text { Suren } \\
\text { in Pro-! } \\
\text { zenten }\end{array}$ & Summe \\
\hline$\theta$ & 77 & 93 & 6 & 7 & 83 \\
\hline 1 bis mehrere & 17 & 27 & 46 & 73 & 63 \\
\hline Summe & 94 & 64 & 52 & 36 & 146 \\
\hline
\end{tabular}

Vereinfachung B der Tabelle XI von Mattauschek und Pilcz.

\begin{tabular}{c|r|r|r|r|r}
\hline Rezidive & $\begin{array}{c}\text { Ohne Behand- } \\
\text { lung } \\
\operatorname{lin}_{\text {in Pro- }}\end{array}$ & $\begin{array}{c}\text { Hg-Behand- } \\
\text { lung } \\
\text { zenten }\end{array}$ & Summe \\
\hline \begin{tabular}{c|r|r|r|r} 
in Pro- \\
zenten
\end{tabular} & \\
\hline 1 bis mehrere & 33 & 50 & 50 & 60 & 83 \\
\hline Summe & 38 & 26 & 108 & 74 & 146
\end{tabular}

Wenn nun tatsächlich die ungenügende Therapie bei den leichten Luesfällen die Ursache der Paralyse wäre, dann müßte unter den Paralytikern, welche an einer rezidivierenden Lues gelitten haben, auch der Prozentsatz der schlecht Behandelten wesentlich größer sein als derjenige der gut und energisch Behandelten. Die Tabellen zeigen uns aber das Gegenteil: Bei der schwereren Lues stehen die Behandelten zu Unbehandelten im umgekehrten Verhältnis wie bei der leichten Lues. Ob man die Tabelle so zusammenzieht, daß Unbehandelte Behandelten gegenüberstehen oder gründlich Behandelte gegenüber nicht oder wenig Behandelten, immer kommt heraus, daß der Prozentsatz der rezidivierenden behandelten Paralytiker wesentlich größer ist als derjenige der behandelten leichten Luesfälle. Ich würde deshalb aus den Tabellen von Matta uschek und Pilez den Schluß ziehen, daß es für die Ent- 
wicklung der Paralyse wesentlich wichtiger ist, ob die Luesinfektion leicht oder schwer verlaufen ist, als ob eine gründliche Behandlung stattgefunden hat oder nicht, ja vielleicht könnte man auf Grund der so gewaltigen Zahlendifferenzen sagen, daß der antiluetischen Behandlung kaum eine prophylaktische Wirkung zukommt. Man könnte ja immerhin einwenden, daß man nicht ausschließen kann, ob sich durch die Behandlung der Prozentsatz nicht doch etwas geändert hat; wenn mir das auch nicht wahrscheinlich erscheint, beweisbar wäre es dann, wenn man berechnen könnte, mit welcher Wahrscheinlichkeit ein behandelter Luetiker mit Rezidiven und mit welcher ein unbehandelter Luetiker mit Rezidiven an Paralyse erkrankt. Eine derartig verarbeitete Statistik existiert àber noch nicht.

Mattauschek und Pilcz haben eine Tabelle (XII) zusammengestellt, aus der sich eine Antwort auf die Frage ergeben soll, ob die vorausgegangene Syphilisbehandlung einen Einfluß auf die Länge des Intervalles zwischen Infektion und Ausbruch der Paralyse erkennen läßt; sie verneinen dies auf Grund dieser Tabelle. Auch dieser Schlußfolgerung möchte ich widersprechen. Tatsächlich kann man aus der Originaltabelle XII selbst nicht viel entnehmen; wenn man aber das Material derselben so umrechnet, daß man den durchschnittlichen Intervall der drei Kolonnen bestimmt, indem man die Zahl der einzelnen Fälle mit der Zahl der entsprechenden Intervalljahre multipliziert, die auf die einzelnen Kolonnen entfallenden Zahlen addiert und durch die Zahl aller Fälle der Kolonnen dividiert, so kommt man zu einem. ganz anderen Resultat. Es findet sich dann, daß der Intervall zwischen Infektion und Paralyse bei Unbehandelten 15,62 Jahre, bei nur einmal mit $\mathrm{Hg}$ Behandelten 13,56 und bei wiederholt Behandelten 15,4 Jahre beträgt, resp. vereinfacht, daß bei unbehandelten Fällen die Paralyse durchschnittlich nach 15,62 Jahren auftritt und bei mit $\mathrm{Hg}$ Behandelten nach 14,36 Jahren. Man ersieht also daraus einen Untersohied von 1,3 Jahren, in dem Sinne, daß die Quecksilberbehandlung die Latenzzeit zwischen Infektion und Paralyse deutlich abkürzt.

Ich möchte also an der Hand der Tabellen von Mattauschek und Pilcz zu einem anderen Schlusse gelangen, als die Autoren selbst. Ich $\mathrm{mu} ß$ daraus entnehmen, daß wenigstens die bisherige Therapie der Lues (Salvarsan nicht eingerechnet) die Paralyseentwicklung nicht hemmt, ja daß sie sie eigentlich um eine Spur beschleunigt. Deswegen dürfte auch der Einwand, daß die mangelhafte Quecksilberbehandlung die Ursachedes hohen Prozentsatzes der metaluetischen Ehemänner paralytischer Frauen ist, abzuweisen sein.

Dieser Schluß von der geringen Wirkung des Quecksilbers erscheint recht befremdend, obzwar dasselbe bereits wiederholt in der Literatur 
behauptet worden ist. Er wird aber weniger befremdend, wenn man die recht verwickelten Verhältnisse der Lues des Zentralnervensystems genauer berücksichtigt. Utber die gute Wirkung des Quecksilbers auf die Hautlues braucht man heute wohl nicht mehr zu diskutieren, ebenso auch nicht über die gute Wirkung auf die Gummen überhaupt; aber gerade unter den Gummen gelten bekanntlich als Ausnahme die Gummen des Zentralnervensystems, die sich dem Quecksilber gegenüber meist recht refraktär verhalten. Die Ursache muß hier wohl in erster Linie in dem Sitz dieser Gummen also dem Nervensystem gesucht werden. Von der Metalues wissen wir, daß sie im allgemeinen vom Quecksilber kaum beeinflußt wird, wir wissen aber noch gar nicht, wann die Metalues beginnt; nicht unwahrscheinlich klingt die Erklärung, daß diejenigen Luesfälle, welche schon in den Frühstadien der Lues eine Pleocytose aufweisen, das Luetikerkontingent darstellen, aus dem die Metaluetiker entstammen; als Ursache der Frühpleocytose der Luetiker muß aber eine frühzeitige luetische Meningitis wohl ganz leichten Grades angenommen werden, so daß folglich diese Frühmeningitis den Urbeginn der Metalues darstellen könnte. Wenn nun das Quecksilber auf die anderen luetischen Produkte im Zentralnervensystem therapeutisch kaum wirkt, dann ist es nicht ausgeschlossen, daß es auch auf diese Frühmeningitis weniger einwirkt, und die geringe Prohibitivwirkung des Quecksilbers gegen die Metalues wäre verständlich. Die Möglichkeit eines derartigen Zusammenhanges läßt sich einstweilen wohl nicht bestreiten, ein klarerer Einblick in diese verwickelten Verhältnisse wird sich aber erst dann gewinnen lassen, bis wir die Entstehungsbedingungen der luetischen Frühmeningitis und deren Verhalten bei der Therapie genauer kennen werden.

Hingegen kann aber noch ein anderer Einwand gemacht werden: es gibt leichte und schwere Luesformen. Die leichten Luesformen neigen mehr zur Paralyse. Aus selbstverständlichen Gründen suchen aber viel eher die schwereren Fälle eine spezialistische Behandlung auf, wogegen sich die leichteren Fälle viel seltener antiluetisch behandeln lassen. Es ist nun recht wahrscheinlich, daß sich unter den Luetikern, welche nicht ärztlich beobachtet wurden - und hierher gehören die Männer der paralytischen Frauen - mehr Fälle von ganz leichter Lues befinden werden, als in dem Materiale einer dermatologischen Klinik. Deshalb könnte man auch erwarten, daß der Prozentsatz der konjugalen Männerparalysen größer sein müsse, als es der Paralysemorbidität bei der ,gewöhnlichen" Lues entspricht und natürlich auch dann, wenn der antiluetischen Behandlung keine besondere prophylaktische Bedeutung zugestanden werden kann. Diesen Einwand kann man natürlich nicht so leicht entkräften, namentlich deshalb nicht, weil er sich direktstatistisch scheinbar in keiner Weise widerlegen läßt. 
Ich habe es dennoch versucht, auf Grund in der Literatur zu anderen Zwecken publizierter Angaben statistische Belege zu diesem Punkte ausfindig zu machen. Ein hiezu recht günstiges Material entstammt der Arbeit von A. Hauptmann: Serologische Untersuchungen von Familien syphilogener Nervenkranken (diese Zeitschrift 8, 1912). Hau ptmann hat sich die Aufgabe gestellt, ganze Familien, aus denen syphilogene Krankheitsfälle stammen, serologisch nach Wassermann zu untersuchen, um auf diesem Wege die eventuell vorhandenen latenten Luesfälle zu eruieren. Von ganz besonderem Interesse für unsere Frage erschien mir die Beobachtung Hauptmanns, daß unter den Frauen von 20 Syphilitischen, die Symptome eines spezifischen Cerebrospinalleidens zeigten, nur eine einzige eine luetische Infektion mit manifesten Symptomen bot, während unter den Ehehälften von 7 Syphilitischen ohne spezifische Cerebrospinalleiden 4 sich befanden, bei denen die luetische Infektion mit manifesten syphilitischen Symptomen einherging. Hauptmann versucht dieses Mißverhältnis in der Art zu erklären, „daß das infizierende Agens, die Spirochäten, durch die Passage durch den Organismus und zwar gerade dann, wenn hierbei das Zentralnervensystem mitbeteiligt ist, so viel an Virulenz verliert, daß es beim Ubergang auf einen zweiten Organismus nicht mehr imstande ist, signifikante Haut- und Schleimhauterkrankungen hervorzurufen, daß dagegen, wenn das Nervensystem bei der Passage frei bleibt, die Infektion sich durch die bekannten Primär- und Sekundärerscheinungen äußert".

Dieser Erklärungsversuch erscheint mir aber nicht richtig zu sein. Man muß dabei vorerst berücksichtigen, daß man es durchweg mit Frauen zu tun hat, die sich wenig beobachten, bei denen leichtere Genitalaffektionen, besonders wenn sie keine Beschwerden machen - und das ist bei den meisten leichteren Primäraffektionen der Fall übersehen werden; ich möchte deshalb den Schluß Hauptmanns, daß die betreffenden Frauen überhaupt keine Primärerscheinungen hatten, in dem Sinne modifizieren, daß die Primärerscheinungen (in welchem Prozentsatz bleibt uns natürlich unklar) nur unscheinbar waren und schnell vorübergingen, so daß sie der Aufmerksamkeit der Frau entgingen. Ich glaube wohl mit größter Wahrscheinlichkeit an. nehmen zu können, daß wenn es sich um ähnliche Infektionsverhältnisse bei Männern gehandelt hätte, wir von denselben viel eher berichtet finden würden, daß der Primäraffekt ganz klein war und sehr bald verschwand, kurzum, daß es sich um die sog. leichte Lues gehandelt hätte.

Wenn wir uns hingegen eine Tabelle aus dem Material von Ha u ptmann in der Weise zusammenstellen, wie es die nebenstehende Tabelle zeigt, so ergibt sich bei Berücksichtigung des gerade Gesagten eine andere Fragestellung. Wir sehen in der Tabelle, daß die Frauen von 17 an Para- 
Tabelle nach dem Material von Hauptmann.

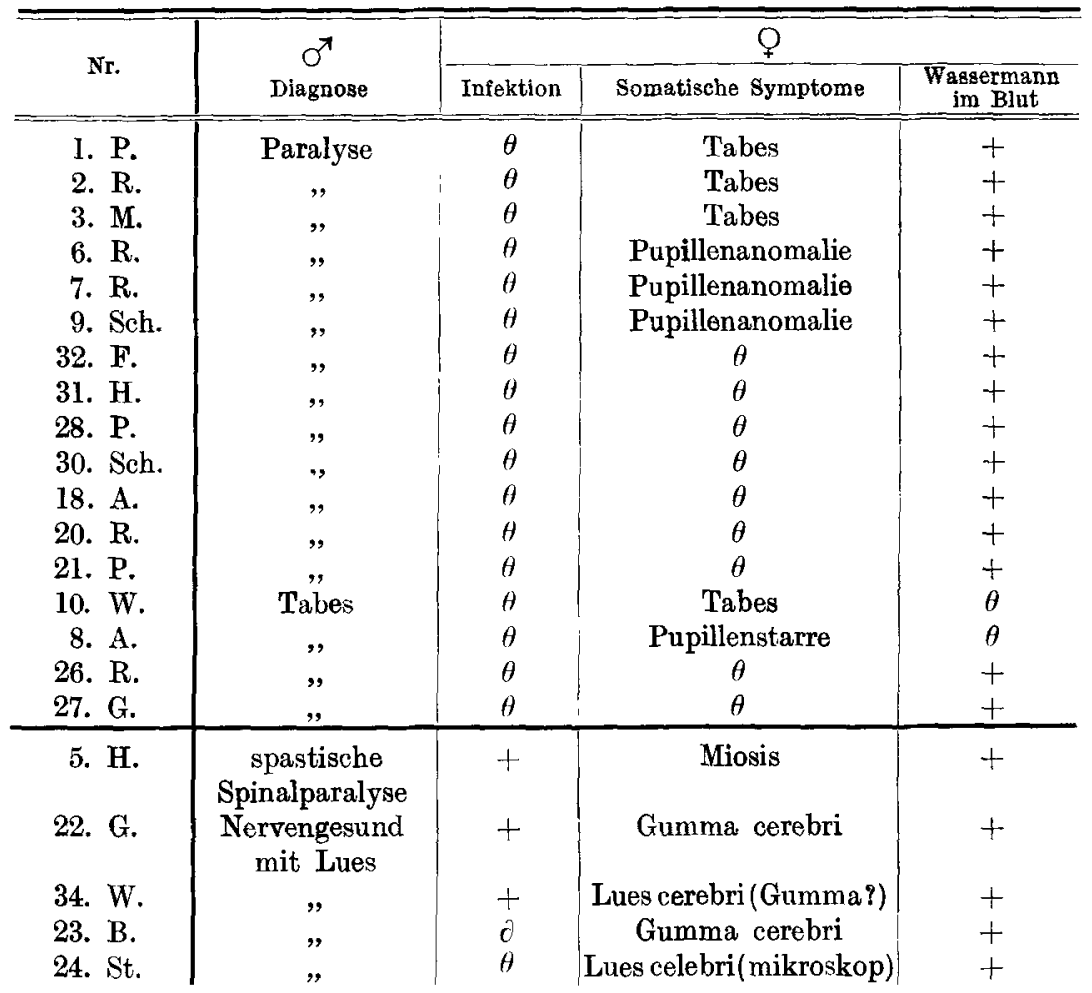

lyse resp. Tabes erkrankten Männern (entweder nach den klinischen Symptomen oder nach der Wasserman nschen Reaktion zu schließen) sicher luetisch infiziert worden sind, alle aber derart, daß sie selbst von einer Infektion nicht wußten. Ein großer Teil dieser Frauen zeigte auch entweder Tabes oder an Tabes resp. Paralyse erinnernde Pupillenstörungen. Wir wissen nun, daß die meisten männlichen Paralyse- (resp. Tabes-)Fälle eine leichteLues durchgemacht haben; diese infizierten ihre Frauen mit einer Lues, die wiederum sehr "leicht" verlaufen ist. Demgegenüber ergibt die Beachtung der unteren 5 Fälle derselben Tabelle ein ganz anderes Resultat. Da handelt es sich um Ehepaare, bei denen $4 \mathrm{mal}$ die Frau und einmal der Mann an Lues cerebrospinalis (meist gummöser Art) erkrankt waren; von den Männern ist überall notiert, daß sie manifest luetisch infiziert waren, und auch bei drei der Frauen ließ sich anamnestisch eine (behandelte) Luesinfektion nachweisen, nur zwei Frauen wußten nichts von manifester Luesinfektion.

Also dieParaly tiker (resp. Tabiker), die meisteine „leich te“ Lues haben, infizieren ihre Frauen mit "leichter" Lues, die in einem auch hier recht auffallend großen Prozentsatz (8 von 17) zu 
tabes- (resp. paralyse-) ähnlichen Symptomen führte, wogegen nichtparalytische Luetiker ihre Frauen mit zum großen Teil manifester Lues infizieren. Wir brauchen also hier nicht anzunehmen, daß erst die von Ha u t mann angenommene Passage durch das Nervensystem die Virulenz des Virus abschwächt, sondern die hier angeführten Männer hatten meist nur eine leichte Lues und diese blieb bei ihrer Utberimpfung auf Ehefrauen ebenfalls leicht. Sie bleibt leicht, führt aber wiederum, wie es auch aus dem Material von Hauptman n ersichtlich ist, mit großem Prozentsatz zur Metalues. Wenn aber die leichte Lues häufig zu Metalues führt, wenn dieselbe als solche unter Beibehalt ung aller ihrer Eigenschaften (erstens leichte Form [quoad Hauterscheinungen], zweitens Neigung zur Bildung von Metalues) ü bertrag bar ist, dann haben wir auch auf diesem Wege den Beweis der Lues nervosa; die "leichte" Lues ist die Lues nervosa.

Dabei ist es aber notwendig, sich klar zu machen, wieweit dieser Begriff der Lues nervosa reicht. Von Erb und anderen ist dies so verstanden worden, daß die Lues nervosa imstande ist, nicht nur in gehäufter Menge Metalues zu erzeugen, sondern luetische Veränderungen des Zentralnervensystems überhaupt, also auch das solitäre Gumma oder eine meningitisch-gummöse Erkrankung des Nervensystems. Von vornherein $\mathrm{mu} ß$ man dagegen ein gewisses Bedenken tragen, da die Paralyse wie auch die Tabes sowohl vom allgemein pathologischen als auch histopathologischen Standpunkt aus etwas wesentlich anderes ist als die bekannten drei Stadien der Lues. Der Paralyse oder der Tabes gleichkommende Erkrankungen gibt es sonst nicht im Körper, dagegen unterscheidet sich das Gumma des Gehirns in gar nichts von dem andersartig lokalisierten Gumma. Deshalb hätte ich ein gewisses Bedenken, wenn eine besondere Form der Lues als Lues nervosa angenommen werden muß, dieselbe auch als wichtigste Ursache der gummösen Erkrankungen des Nervensystems anzunehmen. Ubrigens hat sich auch aus den hier angestellten Utberlegungen ergeben, daß die Lues nervosa, wenn auch vielleicht nur zum Teil, mit der sog. leichten Lues zusammenfällt, von der wir aber wissen, daß gerade sie besonders selten zu Gummen führt, wogegen die ausgebildete tertiäre Lues gegen die Metalues beinahe immunisiert.

So wie sich aber noch schwierigere Fragen auf Grund einer richtigen statistischen Zusammenstellung beantworten lassen, so müßte auch diese Frage statistisch zu erledigen sein; doch bevor wir dazu übergehen, wäre noch einer weiteren Konsequenz zu gedenken, die sich aus dem Bejahen einer Lues nervosa ergibt. Wir fanden nämlich, daß es eine bestimmte Art von Lues gibt, die zu Paralyse und Tabes führt; dieselben sind sicher sehr verwandte Krankheiten, so daß man sie Schwesterkrankheiten nannte; es ergibt sich nun in weiterer Konsequenz die 
Frage, warum der eine eine Paralyse bekommt, der andere eine Tabes, ob nur eine besondere persönliche Disposition als Ursache anzunehmen ist, oder ob auch da Differenzen im Virus anzunehmen oder abzulehnen sind. Alle diese Fragen lassen sich leicht statistisch beantworten, wenn man das bisher bekannte Material konjugaler Fälle von Metalues und cerebrospinaler Lues nach den hier erörterten Gesichtspunkten sorg. fältig zusammenstellt, wozu sich am besten Tabellen eignen, weil man hierbei die verschiedenen Verhältnisse am besten übersieht. Dazu bietet die Arbeit von Fischler: Uber die syphilogenen Erkrankungen des zentralen Nervensystems und über die Frage der Syphilis à virus nerveux (Deutsche Zeitschr. f. Nervenheilk. 28, 1905) ein willkommenes Material. Fischler hat nämlich die bis zum Jahre. 1905 bekannt gewordenen Fälle von konjugaler Lues des Nervensystems zusammengetragen und kritisch besprochen. Die Zusammenstellung der darin enthaltenen konjugalen Fälle (die Ehepaare der Gruppe der familiären Erkrankungen einbezogen) ergibt folgende Verhältnisse:

\begin{tabular}{|c|c|c|}
\hline Beide & Ehegatten hatten Paralyse & $26 \mathrm{~m}$ \\
\hline & , $\quad, \quad$ Tabes & 31 \\
\hline Mann & $\begin{array}{l}\text { tabisch, Frau paralytisch } \\
\text { paralytisch, Frau tabisch }\end{array}$ & \\
\hline Beide & Ehegatten Lues cerebrospinalis & 4 \\
\hline Mann & Tabes, Frau Lues cerebrospinali & \\
\hline & Paralyse, , $\quad, \quad \quad$, & \\
\hline
\end{tabular}

Aus der Gruppe der familiären Erkrankungen ergibt sich:

Einer der Eltern Paralyse, das Kind Paralyse $12 \mathrm{mal}$,

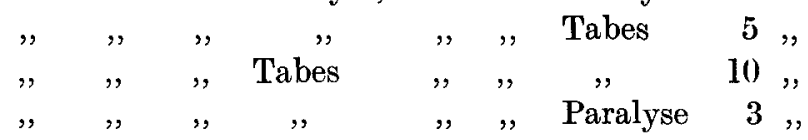

Vorerst zeigen sich interessante Verhältnis für die Lues cerebrospinalis (meist gummosa gemeint). Inter 6 Ehepaaren leiden 4 mal beide Ehehälften an Lues cerebrospinalis und nur zweimal finden wir bei einem Ehegatten Lues cerebrospinalis, beim anderen Paralyse oder Tabes. Gewiß sind 6 Fälle noch recht wenig, viel zu wenig, als daß man große allgemeine Schlüsse daraus ziehen könnte, immerhin bestätigen sie die vorhin erhobenen Bedenken.

Vielleicht noch interessantere Ergebnisse ergaben sich in der zweiten Frage, die Tabes und Paralyse betreffend. Wenn man die Fälle der Fischlerschen Arbeit danach zusammenstellt, ob beide Ehehälften an derselben Form der Metalues (Tabes-Tabes, Paralyse-Paralyse) litten oder Mann und Frau je eine ungleichartige Metalues (Tabes - Paralyse) hatten, und in gleicher Weise auch die Fälle von Metalues bei Eltern und Kind dazu zählt, so ergibt sich folgendes Verhältnis: 
Gleichartige Metalues (bei Familienangehörigen) $79 \mathrm{mal}$,

Ungleichartige ," " " 27 ,

Das ist ein Verhältnis, das wohl zu denken gibt. Um das Material zu vergrößern, habe ich noch das in den nach 1905 erschienenen Publikationen von Mönkemöller, Junius und Arndt und Hannard et $\mathrm{Ga} \mathrm{yet}^{1}$ ) enthaltene Material konjugaler Lues hinzugerechnet, sowie das von mir bisher beobachtete Material von konjugaler und familiärer Metalues. Dann ergibt sich folgende Tabelle:

\begin{tabular}{r||c|c}
\hline \multirow{2}{*}{ Provenienz des Materials } & \multicolumn{2}{|c}{ Metalues } \\
\hline & Gleichartig & Ungleichartig \\
\hline Fischler . . . . . . . & 79 & 27 \\
Mönkemöller . . . . . & 15 & 3 \\
Junius und Arndt. . . & 31 & 7 \\
Hannard et Gayet. . . & 21 & 4 \\
Eigenes Material. . . . & 12 & 4 \\
\hline Summa & 158 & 45 \\
in Prozenten & $78 \%$ & $22 \%$
\end{tabular}

Es zeigt sich also, daß die konjugale gleichartige Metalues mehr als dreimal häufiger vorkommt als die ungleichartige. Bei dem so großen Material ist der Zufall ganz auszuschließen, um so mehr als sich dasselbe Verhältnis auch aus dem Material der einzelnen hier erwähnten Autoren ergibt. Und die Erklärung? Kaum etwas anderes als die Annahme, daß es auch beim Virus der Lues nervosa zwei Unterarten geben müsse, von denen das eine prozentuell häufiger zur Tabes, das andere zur Paralyse führt, wobei aber ausdrücklich wieder betont werden muß, daß dies nicht immer, sondern nur prozentuell häufiger geschieht. Dabei muß die von Mendel bereits gefundene Regel berücksichtigt werden, daß bei ungleichartiger konjugaler Metalues der Mann viel häufiger paralytisch ist und die Frau dagegen tabisch. Wenn man das hier berücksichtigte Material von 167 Ehepaaren, i. e. 334 Einzelfällen, so zusammenstellt, daß man berechnet, wieviel Frauen und wieviel Männer an Paralyse resp. Tabes (bei konjugaler Metalues natürlich) erkranken, dann ergibt sich folgende Tabelle:

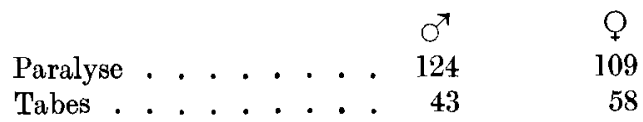

oder noch besser, wenn man in gleicher Weise die 37 an ungleichartiger Metalues erkrankten Ehepaare (74 Einzelfälle) zusammenstellt:

1) De la paralysie générale et de la Tabo-Paralysie conjugale. Annales méd.psychol. 1911 . 


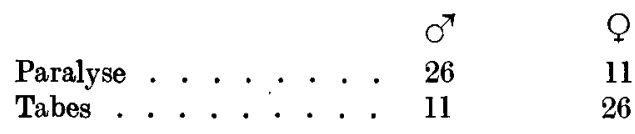

Man ersieht daraus, daß bei den ungleichartig metaluetischen Ehepaaren die Tabes bei Frauen mehr als zweimal so häufig ist als bei Männern, was für die Paralyse in umgekehrter Weise gilt, d. h. daß neben den erschlossenen Differenzen des Tabes- und Paralysevirus noch eine besondere Disposition anzunehmen ist, die sich wenigstens für die Geschlechter so verhält, daß die Frauen mehr zur Tabes, die Männer mehr zur Paralyse neigen.

Es dürfte wohl selbstverständlich erscheinen, daß, so wie man für das Paralyse- und für das Tabesvirus beweisen konnte, daß es nicht immer dieselbe Form der Metalues hervorruft, man auch für die Lues nervosa überhaupt auch nicht annehmen kann, daß sie immer zu einer Metalues führen muß; dabei möchte ich betonen, daß es vielleicht auch verschiedene Abstufungen geben kann, so daß das eine Virus prozentuell mehr Metalues verursacht, das andere weniger (so in der Glasbläserepidemie von Brosius in $80 \%$ ), ebenso wie man auch nicht ausschließen kann, daß hin und wieder eine gewöhnliche Lues ebenfalls zu einer Metalues führen kann, wenn auch, wie es aus unseren Zusammenstellungen ersichtlich ist, wesentlich seltener. Wenn ein trivialer Vergleich gestattet ist, verhalten sich diese zwei Virusarten zueinander wie etwa eine gezogene Pistole zu einer nichtgezogenen. Mit der gezogenen Pistole trifft man viel besser und leichter, mit der nichtgezogenen viel schlechter, man kann aber nicht sagen, da $B$ man mit der gezogenen immer trifft und mit der nichtgezogenen nie; es hängt von der Größe und Entfernung des Zieles ab und überdies auch von der Geschicklichkeit des Schützen.

Der Begriff der Lues nervosa, als Folge einer besonderen Varietät der Spirochäte, widerspricht nun in keiner Weise unseren allgemeinpathologischen Erfahrungen. So weiß man, daß gewisse Epidemien (Typhus, Influenza) einmal sehr leicht, das andere Mal sehr schwer verlaufen, die Bubonenpest geht in manchen Epidemien häufiger als Lungenpest einher als in anderen und der Streptokokkus erzeugt manchmal Erysipel, in welcher Eigenschaft er auch verimpfbar ist (Erysipelendemien).

Für unser Kapitel ist aber von ganz besonderer Wichtigkeit eine Beobachtung Spielmeyers ${ }^{1}$ ) bei Impfversuchen mit dem Trypanosoma Brucei. Durch mehrfache Passagen durch weiße Mäuse und Kaninchen erhielt S pielmeyer einen Stamm, der Hunde nicht mehr wie früher in 5-8 Tagen tötete, sondern erst nach 10-15 Wochen; die mit diesem abgeschwächten Stamm infizierten Hunde zeigten in

1) Therapie der progressiven Paralyse. Archiv f. Psych. 50. 
etwa $70-80 \%$ systematische degenerative Veränderungen an den Hinterwurzeln und den Trigeminuswurzeln, welche einer menschlichen Tabes nicht unähnlich waren. In einem Zeitraum von 10-12 Monaten verlor aber dieser das Nervensystem so eigenartig angreifende Stamm die Eigenschaft, nervöse Erkrankungen beim Hund zu erzeugen, obzwar sich seine sonstige Virulenz gar nicht geändert hatte; auch jetzt tötete er Hunde in 10-15 Wochen. Spielmeyer weist mit Recht darauf hin, daß wir darin nur eine besondere und vorübergehende Eigenschaft des Virus im Sinne einer Affinität zum Nervensystem sehen müssen und schließt daraus, daß so wie wir hier von einer Art Tripanosomiasis à virus nerveux sprechen müssen, wir dies auch bei der Syphilis mit Recht annehmen können, um so mehr, als es sich um Erkrankungen handelt, deren Erreger immerhin gewisse verwandte Eigenschaften haben.

Spielmeyer weist auch darauf hin, daB man auf Grund seiner Erfahrungen mit dem Trypanosoma, das plötzlich die nervenkrankmachendeEigenschaft bekommt und sie auch so plötzlich verliert, auch erklären könnte, warum die Paralyse nach längst bekannter und vorkommender Lues plötzlich aufgetreten ist; man könnte auch deshalb erwarten, daß auch das Luesvirus seine neurotropen Eigenschaften verlieren könnte, so daß dann die Paralyse aufhören müßte. Selbstverständlich ist diese Möglichkeit nicht beweisbar. Immerhin möchte ich darauf hinweisen, daß eine Notiz in der Arbeit von Mattauschek und Pilcz unter Umständen für dieselbe sprechen könnte. Mattauschek und Pilcz haben bei Behandlung der Frage, ob die antiluetische Behandlung die spätere Paralyse verhüten kann, unter anderem auch folgendes erwogen: Früher wurden die Luetiker schlechter und weniger behandelt als jetzt; wenn es richtig ist, daß die antiluetische Behandlung die Paralyse verhüten kann, dann müßte in der neueren Zeit die Paralyse prozentuell seltener geworden sein. Mattauschek und Pilcz haben deshalb die Prozente für Paralyse bei den Luetikern in den Jahren 1880-1884 und 1895-1899 gesondert berechnet und fanden bei den älteren Jahrgängen $9,72 \%$, bei den jüngeren $3,25 \%$. Das spräche also dafür, daß die antiluetische Behandlung die Paralyse doch wesentlich einschränken könne. Nun habe ich aber auf Grund der Tabellen derselben Autoren darauf hingewiesen, daß eine wesentliche Beeinflussung der Metalues durch eine vorhergehende antiluetische Therapie nicht recht (zumindest in diesem Maße) wahrscheinlich erscheint; wenn dies richtig wäre, dann könnte man das Mißverhältnis $9,72: 3,25 \%$ ev. auch mit einer Änderung der neurotoxischen Eigenschaften des Virus erklären. Ich bin mir aber darüber klar, daß dieser Schluß viel zu viel hypothetisch ist, da man aus keiner der Tabellen herauslesen kann, ob die antiluetische Behandlung die Paralyse nur wenig oder gar nicht einzuschränken imstande ist. 
Gegen die Annahme einer Lues nervosa hat sich besonders scharf

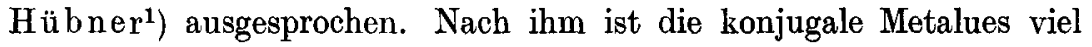
zu selten, als daß sie die Annahme eines besonderen nervösen Virus angezeigt erscheinen lassen würde, und die konjugale Metalues wird einfach als Folge eines Zufalls erklärt. Meine Statistik widerlegt diesen Einwand. Ferner legt Hübner dem bereits längst bekannten Umstande eine besondere Bedeutung bei, daß nämlich in Familien mit mehreren Metaluesfällen nicht alle Familienmitglieder metaluetisch werden, sondern $d a \beta$ einzelne derselben, trotzdem sie luetisch wurden, dennoch ein gesundes Nervensystem behielten. Das würde nur dann dem Begriff einer Lues nervosa widersprechen, wenn man sich vorgestellt hätte, daß eine derartige Infektion immer zu einer Alteration des Nervensystems führen müßte. So viel mir aber aus der Literatur bekannt ist, hat niemand die Lues nervosa in dieser Weise aufgefaßt, und wenn, dann wäre es unvorsichtig und falsch gewesen. Hübner macht überdies noch einen weiteren Einwand. Einige Gegner der Lues ätiologie der Paralyse haben nämlich immer als Haupteinwand angeführt, daß die Paralyse bei den Prostituierten viel zu selten vorkomme. Hübner hat nun das Frauenmaterial der Irrenanstalt zu Herzberge bei Berlin von dem Standpunkte aus gesichtet und findet unter den Prostituierten resp. gewesenen Prostituierten (und auf letzteres ist ein besonderes Gewicht zu legen) 20,9\% Paralysen, unte den andern Frauen aber nur 13,5\%. Bei Ausdehnung seiner Beobachtung auf ein Lazarettmaterial findet er unter 179 Prostituierten $38,4 \%$ Paralyse, Tabes und Lues cerebrospinalis. Das ist natürlich ein ungemein hoher Prozentsatz, und $\mathrm{Hüb}$ ner findet dies unvereinbar mit der Lues-nervosaTheorie, da man sonst nicht annehmen könne, daß die Prostituierten häufiger eine Lues nervosa haben sollten als andere Menschen. Dieser Schluß beruht aber auf einem Irrtum, denn die Berechnung der Prozente auf die Art, wie es Hübner tut, beseitigt zwar endgültig den früheren Einwand, da $B$ die Paralyse unter Prostituierten selten sei, sie beweist uns aber nur, da $ß$ sich unter Geisteskranken oder siechen Frauen prozentuell viel weniger Paralysen befinden als unter denjenigen Prostituierten, die sich - und darauf ist das Hauptgewicht zu legen auch wegen Krankheit in Irrenanstalten oder Siechenhäusern befinden. Man kann aber gar nicht daraus entnehmen, wie häufig (prozentuell) syphilitische Prostituierte überhaupt an Paralyse erkranken. Hübner hat eben nicht berücksichtigt, daß man zu einer so allgemein gehaltenen Berechnung ein ganz anderes Material braucht; da muß man auch die gesunden Prostituierten einbeziehen, die keine Ursache haben, eine Irrenanstalt oder ein Siechenhaus aufzusuchen. Für die Frage der Lues nervosa kommt also auch dieser Einwand $\mathrm{Hüb}$ ners nicht in Betracht.

$\left.{ }^{1}\right)$ Berliner klin. Wochenschr. 1906. 
Man ersieht aus all dem, wie kompliziert die Art einer derartigen statistischen Beweisführung ist, daß sie sich komplizierter gestaltet hat, als es in den bisherigen statistischen Bearbeitungen der Fall war, und zwar wegen der vielen wichtigen Nebenfragen. Die jeweilige Hauptfrage, so einfach sie zuerst erscheint, wird bei näherem Betrachten viel schwieriger, die ursprünglich geschlossene Masse des statistischen Materials wird zerspalten und erst nach Berücksichtigung aller Momente und Nebenmomente, manchmal mit recht präziser, manchmal wieder mit nur approximativer Annäherung an die Idealverhältnisse, schreitet man zu den notwendigen Schlußfolgerungen. Wenn ich auch mir dessen voll bewußt bin, daß die Größe und Art des hier verwendeten Materiales doch nicht ganz den notwendigen Ansprüchen gerecht wird, so glaube ich doch, daß die Frage der Lues nervosa nicht auf die bisher durchgeführte Art zu erledigen ist, sondern auf dem hier eingeschlagenen Wege. Wenn auch ein entsprechend zusammengetragenes größeres und vielleicht besseres Material noch manchen der hier gezogenen Schlüsse ändern kann, soviel erscheint mir bereits sicher zu sein, daß wir die Frage, ob es eine Lues nervosa gibt, bejahen müssen. 